\title{
Role of oral hypoglycemic drugs on inflammatory condition associated with type 2 diabetes mellitus
}

\begin{abstract}
Pathogenesis of type 2 is observed to be closely associated with acute phase response which is predominately cytokine-mediated. Maximum up to date literature used to depicts that diabetes and secondary complications with chronic inflammation leads to immunological changes shown by altered levels of cytokines and chemokines, changes in the numbers and activation states of various leukocyte populations, apoptosis, and fibrosis during diabetes. $\alpha 1-$ antitrypsin, $\alpha 1-$ acid glycoprotein ceruloplasmin and fibrinogen used to consider to be very sensitive and reliable parameters in group of acute phase proteins. In this present study I try to test this hypothesis by estimating circulating acute phase proteins in freshly diagnosed type 2(T-2) as well as the alteration of levels of these inflammatory markers in type 2 diabetic patients under oral hypoglycemic drugs for duration of at least five years. Thirty normal controls to match the age and sex of the test groups were also studied. The level of this parameter was also correlated with their random plasma glucose values and BMI. The value of all these parameter significantly elevated in the T-2 patients $(p<.00001)$ in comparison with the controls. Out of these four parameters levels of $\alpha 1$ - antitrypsin, $\alpha 1$ - acid glycoprotein reduced significantly after treatment by oral hypoglycemic drugs. Interestingly in either of the types, no correlation was found with the degree of hyperglycemia or BMI. By the above results and findings it can be definitely postulated that oral hypoglycemic drugs have definite role to reduce glycemia status as usual. But the role of the oral hypoglycemic drug to reduce the inflammatory state cannot be ruled out.
\end{abstract}

Keywords: acute phase proteins, acute phase reactants, oral hypoglycemic drugs, inflammatory condition, chronic low grade inflammation, diabetes mellitus
Volume 5 Issue 2 - 2018

\section{Shamim Shaikh Mohiuddin,' Poornima Manjrekar $^{2}$}

'Department of Biochemistry, Imam Abdulrahman Bin Faisal University, Saudi Arabia

${ }^{2}$ Department of Biochemistry, Manipal University, India

Correspondence: Shamim Shaikh Mohiuddin, Assistant Professor, Department of Biochemistry, College of Medicine. Imam Abdulrahman Bin Faisal University, PO box- 1982. Dammam- 3|44I, Kingdom of Saudi Arabia, Tel + 00966562539936;

Emailsmohiuddin@iau.edu.sa, ravi94II4@gmail.com

Received: February 17, 2018 | Published: April 23, 2018

\section{Introduction}

Diabetes Mellitus is one of the most common major public health problems having worldwide distribution with a prevalence of 382 million human cases, and the incidence is expected to increase to 592 million by 2035. According to the Centers for Disease Control, present trends of diabetes incidence suggest one in three Americans will be diagnosed with diabetes by the year $2050 .{ }^{1}$ The vast majority of diabetes patients $(90 \%-95 \%)$ suffer from type 2 DM (T2DM). Depending on the etiology of diabetes mellitus, factors contributing to hyperglycemia may include; reduced insulin secretion, decreased glucose usage and increased glucose production. Although hyperglycemia is the main characteristic of all form of diabetes mellitus, the pathogenic mechanism by which hyperglycemia arises differs widely. Some forms of Diabetes mellitus are characterized by an absolute insulin deficiency or a genetic defect leading to defective insulin secretion; where as other forms share insulin resistance as their underlying etiology. ${ }^{2}$ Recently, there in increasing evidence that an ongoing cytokine induced acute phase response which is sometimes called low grade inflammation, but part of a widespread activation of the innate immune system, is closely involved in the pathogenesis of type 2 diabetes mellitus and associated complications such as dyslipidemia and atherosclerosis. Current literature recognizes that chronic low-grade subclinical inflammation is a part of insulin resistance and strongly related to the features of metabolic syndrome..$^{3-5}$ In addition; inflammatory processes are also involved in the micro vascular complications of diabetes including diabetic nephropathy and retinopathy. 9 Inflammatory factors, which play a critical role in the development of atherothrombosis, are often found to be at elevated levels in patients suffering from diabetes. Elevated circulatory inflammatory markers such as C-reactive protein and interleukin- 6 predict the development of type 2 Diabetes mellitus and several drugs with anti-inflammatory properties both lower both acute phase reactants and glycemia and possibility decrease the risk of developing type 2 diabetes mellitus. Age, inactivity, certain dietary components, smoking, psychological stress and low birth weight are among the risk factors for type 2 diabetes mellitus, which are also known to be associated with activated innate immunity. Activated immunity may be the common antecedent of developing type 2 diabetes mellitus. ${ }^{6}$ Other features of type 2 diabetes mellitus such as fatigue, sleep disturbance and depression are likely to be at least partly due to hypercytokinemia and activated innate immunity. This recent explosion of interest in the notion that chronic low grade inflammation and activation of the innate immune system are closely involved in the pathogenesis of type 2 diabetes mellitus was first proposed in 1997-987 several studies after that have shown that circulating markers of inflammation, acute phase reactants or interleukin-6 (IL-6) are strong predictors of the development of type 2 diabetes. ${ }^{8,9}$ The role of acute phase reactants in the development of type 1 diabetes mellitus is not very clear. For the management of Type 2 Diabetes Mellitus, many approach have been investigated which can mainly target to reduce inflammation but no approach concluded as successful. ${ }^{10,11}$ The current pharmacotherapy of Type 2 Diabetes Mellitus is derived from the ominous octet concept described by 
DeFronzo. ${ }^{12,13}$ An adaptive pharmacotherapy plan used to adopted due to progressive nature of type 2 diabetes mellitus which depends on a combination of medications, nutrition management, and exercise. But only by strict control of blood glucose, the prevention and betterment of Type 2 Diabetes Mellitus conditions may not merely lie. That's why the anti-inflammatory characteristics of antidiabetic agents and the inflammatory process that can evoke Type 2 Diabetes Mellitus are concerned as a modern new ideology. Current literatures show an association of diabetes and secondary complications with chronic inflammation. Evidence of these immunological changes include altered levels of cytokines and chemokines, changes in the numbers and activation states of various leukocyte populations, apoptosis, and fibrosis during diabetes. Therefore, treatment of diabetes and its complications may include pharmacological strategies to reduce inflammation. Apart from anti-inflammatory drugs, various hypoglycemic agents have also been found to reduce inflammation that could contribute to improved outcomes. Extensive studies have been carried out with thiazolidinediones (peroxisome proliferatoractivated receptor- $\gamma$ agonist), dipeptidyl peptidase- 4 inhibitors, and metformin (AMP-activated protein kinase activator) with each of these classes of compounds showing moderate-to-strong anti-inflammatory action. Sulfonylureas and alpha glucosidase inhibitors appeared to exert modest effects, while the injectable agents, insulin and glucagon-like peptide-1 receptor agonists, may improve secondary complications due to their anti-inflammatory potential. Currently, there is a lack of clinical data on anti-inflammatory effects of sodiumglucose co transporter type 2 inhibitors. Nevertheless, for all these glucose-lowering agents, it is essential to distinguish between antiinflammatory effects resulting from better glucose control and effects related to intrinsic anti-inflammatory actions of the pharmacological agents. $^{14}$

\section{Material and methods}

\section{Participants}

Following three groups of subjects were selected for present study. Subjects were selected from various private clinics hospitals in Mangalore, India.

a. 25 newly diagnosed untreated type 2 diabetes mellitus patients within the age limit of 30-60 years

b. 25 type 2 diabetes mellitus patients who are under treatment of oral hypoglycemic drugs for at least 5 yrs between the age limit of 30-60 yrs.

c. 30 nondiabetic healthy controls.

Height and weight of all subjects were recorded and body mass index was calculated. None of the ninety two volunteers were alcoholics or smokers. The participants did not suffer from chronic inflammatory diseases like asthma, chronic bronchitis, and rheumatoid arthritis as was ascertained by clinical history. Informed consent was taken from the individual subjects prior to blood collection. The study was approved by institutional ethical committee.

\section{Materials}

$5 \mathrm{ml}$ blood was collected in plain bottle, $2 \mathrm{ml}$ in EDTA bottle and $1 \mathrm{ml}$ in fluoride bottle. Blood was taken from antecubital vein of the subjects.

\section{Methods}

The following methods were followed for determination of each of the parameters.

Estimation of blood glucose: Blood glucose was estimated with Agappe manual diagnostic kit by GOD-POD methodology. The enzymatic procedure of Trinder $\mathrm{P}$ was followed Glucose is oxidized by glucose oxidase to gluconic acid and $\mathrm{H}_{2} \mathrm{O}_{2}$. Hydrogen peroxide in presence of phenol and 4-amino antipyrine is acted upon by peroxidase to form red quinine. The absorbance of colored compound was measured at $532 \mathrm{~nm} .{ }^{15}$

Estimaion of plasma fibrinogen: Fibrinogen assay in plasma was carried out by Biuret method. Fibrinogen present in the plasma is converted to fibrin in presence of calcium chloride. The fibrin clot formed is collected and then digested with $\mathrm{NaOH}$. Protein content of the clot is determined using a red filter. ${ }^{16}$

Estimation of serum ceruloplasmin: Ceruloplasmin assay in serum was carried out by the method of Sunderman FW et al. ${ }^{17}$ At $\mathrm{pH}$ 5.4 , ceruloplasmin catalyses the oxidation of PPD to yield a coloured product which is believed to correspond either to Bandrowski's base or to Weurster's red. The rate of formation of coloured oxidized product is proportional to the concentration of ceruloplasmin, if, a correction is made for the nonenzymatic oxidation of PPD. Therefore simultaneous assay were carried out with and without sodium azide, which inhibited the enzymatic oxidation of PPD. The difference between the results of the two assays is proportional to ceruloplasmin concentration. ${ }^{17}$

Estimation of serum $\alpha 1-$ antitrypsin: $\alpha-1$ antitrypsin assay in serum was carried out by the modified method described by Sundaresh et al. ${ }^{18}$ The Proteolytic enzyme trypsin hydrolyses casein, with the formation of smaller peptides. The enzyme reaction after suitable interval of time is arrested by the addition of TCA which precipitated the protein, but the peptides are soluble in the acid. The TCA soluble fragments are a measure of Proteolytic activity of the enzyme. When the inhibitor is added to the preincubated mixture, it prevents the release of peptides by the Proteolytic enzymes. Thus the estimation of TCA soluble components in the presence and absence of inhibitor is a measure of inhibitory activity against Proteolytic enzymes. The TCA soluble fragments are analyzed by the method of Lowry the final colour formed is a result of Biuret reaction of the peptides with copper ions in alkali and reduction of the phosphomolybdic acid reagent by the presence of tyrosine and Tryptophan which are present in the treated peptides. ${ }^{18}$

Estimation of $\alpha-1$ acid glycoprotein: $\alpha-1$ acid glycoprotein assay in serum was carried out by the method of Winzler RJ et al. ${ }^{19}$ After removing heat coagulable proteins with perchloric acid, the orosomucoid which remains in the solution is precipitated by phosphotungstic acid and estimated by determining it carbohydrate content by reaction with an orcinol-sulphuric acid reagent, or its nitrogen by Kjeldahl nesslerization or its tyrosine content using Folin Ciocalteau reagent. The last of these is employed in the technique given below. ${ }^{19}$

\section{Statistics}

The data was analyzed by the students't test and the ANOVA test. Pearson's coefficient was applied for co relational analysis 


\section{Result}

The anthropometric data of the subjects participated in the study are presented in Table 1. As comparison to control group (Group III) values of BMI and age of patients of newly diagnosed type 2 patients (Group I) and patients of diabetes mellitus under treatment by oral hypoglycemic drug (Group II) were chosen those of almost closely related (Table 2). Comparison of values obtained in all parameters of Group I and Group III patients are shown in Table 3. As compared to the Control group the values of all parameters of Group I were very highly elevated as in shown by the $p$ values of $<0.0001$ in case of all parameters. By comparison Group II with control group (Group III); RBS, $\alpha 1$-antitrypsin and fibrinogen values were very highly

Table 1 The anthropometric data of the subjects participated in the study elevated in Group II as is shown by the $\mathrm{p}$ values of $<0.0001$ for all parameters. Values of $\alpha 1$-acid glycoprotein were significantly low in Group II as compared to control group as shown by $\mathrm{p}$ value of 0.005 . The difference in ceruloplasmin values did not show any statistical significance (Table 4). As compared with Group II the values of $\alpha 1$ acid glycoprotein and ceruloplasmin in Group I were very highly significant as shown by $\mathrm{p}$ value $<0.0001$ in both these parameters. Values of $\alpha 1$-antitrypsin were significant in group I as compared with group II as shown by $\mathrm{p}$ value 0.03 . No statistical significance was obtained regarding RBS and fibrinogen values between group I and group II patients (Table 5). The RBS values did not show any positively correlation with any of the acute phase proteins in all the groups (Table 6).

\begin{tabular}{|c|c|c|c|}
\hline & Group I $(n=25)($ Mean $\pm S D)$ & Group II $(n=25)($ Mean $\pm S D)$ & Group III $(n=30)($ Mean \pm SD $)$ \\
\hline Age (yrs) & $48.22 \pm 7.11$ & $51.32 \pm 7.56$ & $44.97 \pm 15.06$ \\
\hline BMI & $24.03 \pm 1.46$ & $24.20 \pm 2.40$ & $21.75 \pm 2.27$ \\
\hline
\end{tabular}

Group I, Type 2 diabetes mellitus patient (newly diagnosed); Group II, Type 2 diabetes mellitus patient (under treatment for at least 5 years); Group III, Control; $\mathrm{n}$, number of subjects; SD, Standard Deviation; BMI, Body Mass Index

Table 2 Compares the mean values of all parameters between groups

\begin{tabular}{|c|c|c|c|c|c|c|}
\hline Group & $\mathbf{n}$ & $\begin{array}{l}\text { RBS (mg/dl) } \\
\text { Mean } \pm S D\end{array}$ & $\begin{array}{l}\alpha-I \text { antitrypsin } \\
(\mathrm{mg} / \mathrm{dl}) \text { Mean } \pm S D\end{array}$ & $\begin{array}{l}\alpha-1 \text { acid } \\
\text { glycoprotein }(\mathrm{mg} / \\
\text { dl) Mean } \pm S D\end{array}$ & $\begin{array}{l}\text { Ceruloplasmin }(\mathrm{mg} / \mathrm{dl}) \\
\text { Mean } \pm \text { SD }\end{array}$ & $\begin{array}{l}\text { Fibrinogen (mg/ } \\
\text { dl) Mean } \pm S D\end{array}$ \\
\hline Group I & 25 & $193.26 \pm 35.30$ & $562.16 \pm 63.00$ & $181.93 \pm 31.94$ & $45.05 \pm 9.03$ & $572.25 \pm 82.26$ \\
\hline Group II & 25 & $|93.6| \pm 33.65$ & $519.38 \pm 47.80$ & $87.10 \pm 17.69$ & $25.73 \pm 9.94$ & $581.74 \pm 79.09$ \\
\hline Group III & 30 & $94.20 \pm 7.00$ & $350.48 \pm 114.07$ & $103.41 \pm 22.13$ & $26.95 \pm 4.10$ & $335.34 \pm 42.19$ \\
\hline
\end{tabular}

Group I, Type 2 diabetes mellitus patient (newly diagnosed); Group II, Type 2 diabetes mellitus patient (under treatment for at least 5 years); Group III, Control; $\mathrm{n}$, number of subjects; SD, Standard Deviation; RBS, Random Blood Sugar

Table 3 Comparison of values obtained in all parameters of Group I and Group III patients

\begin{tabular}{|c|c|c|c|}
\hline Parameters & Group I $(n=25)$ Mean $\pm S D$ & Group III $(n=30)$ Mean \pm SD & $p$ value \\
\hline RBS ( mg/dl) & $193.26 \pm 35.30$ & $94.20 \pm 7.00$ & $<0.0001^{*}$ \\
\hline$\alpha \mathrm{I}$-antitrypsin(mg/dl) & $562.16 \pm 63.00$ & $350.48 \pm 1 \mid 4.07$ & $<0.0001^{*}$ \\
\hline$\alpha \mathrm{l}$-acid glycoprotein $(\mathrm{mg} / \mathrm{dl})$ & $181.93 \pm 31.94$ & $103.41 \pm 22.13$ & $<0.0001^{*}$ \\
\hline Ceruloplasmin (mg/dl) & $45.05 \pm 9.03$ & $26.95 \pm 4.10$ & $<0.0001^{*}$ \\
\hline Fibrinogen (mg/dl) & $572.25 \pm 82.26$ & $335.34 \pm 42.19$ & $<0.000 \mathrm{I}^{*}$ \\
\hline
\end{tabular}

n, number of subjects; *, statistically significant, Group I, Newly diagnosed type II patients; Group III, Control group

Table 4 Comparison of values obtained in all parameters of Group II and Group III patients

\begin{tabular}{lllc}
\hline Parameters & Group II $(\mathbf{n}=\mathbf{2 5})$ Mean \pm SD & Group III $(\mathbf{n}=\mathbf{3 0})$ Mean \pm SD & p value \\
\hline RBS $(\mathrm{mg} / \mathrm{dl})$ & $193.61 \pm 33.65$ & $94.20 \pm 7.00$ & $<0.0001^{*}$ \\
$\alpha 1$-antitrypsin(mg/dl) & $519.38 \pm 47.80$ & $350.48 \pm 114.07$ & $<0.0001^{*}$ \\
$\alpha 1$-acid glycoprotein(mg/dl) & $87.10 \pm 17.69$ & $103.41 \pm 22.13$ & 0.005 \\
Ceruloplasmin $(\mathrm{mg} / \mathrm{dl})$ & $25.73 \pm 9.94$ & $26.95 \pm 4.10$ & 0.55 \\
\hline
\end{tabular}

n, number of subjects; *, statistically significant, Group II, Type II diabetic patients under treatment, Group III, Control group

Table 5 Comparison of values obtained in all parameters in Group I and Group II

\begin{tabular}{llll}
\hline Parameters & Group I (n=25) Mean \pm SD & Group II (n=25) Mean \pm SD & p value \\
\hline RBS $(\mathrm{mg} / \mathrm{dl})$ & $193.26 \pm 35.30$ & $193.61 \pm 33.65$ & 0.972 \\
$\alpha 1$-antitrypsin(mg/dl) & $562.16 \pm 63.00$ & $519.38 \pm 47.80$ & 0.03 \\
$\alpha 1$-acid glycoprotein(mg/dl) & $181.93 \pm 31.94$ & $87.10 \pm 17.69$ & $<0.0001^{*}$ \\
\hline
\end{tabular}

n, number of subjects; *, statistically significant, Group II, Type II diabetic patients under treatment, Group II, Type 2 patients under treatment 
Table 6 Correlation of RBS with various acute phase proteins in all the groups (r value)

\begin{tabular}{llll}
\hline Parameters & Group I & Group II & Group III \\
\hline$\alpha 1$-antitrypsin & -0.61 & -0.01 & 0.06 \\
$\alpha 1$-acid glycoprotein & -0.15 & -0.03 & -0.46 \\
Ceruloplasmin & -0.15 & 0.14 & 0.12 \\
Fibrinogen & 0.27 & -0.23 & -0.43 \\
\hline
\end{tabular}

\section{Discussion}

The exact effect of inflammatory cytokines on glucose metabolism in humans is still unclear. The followings are the possibly mechanisms of activated innate immunity in type 2 diabetes mellitus. Innate immunity may give rise to the features of type 2 diabetes, including cytokine induced insulin resistance and impaired insulin secretion, increased capillary permeability and microalbuminuria, dyslipidemia, hypercortisolemia, hypertension, central obesity and a hypercoagulent state. Cytokines such as tumor necrosis factor $\alpha(\mathrm{TNF} \alpha)$ can cause insulin resistance by activation of the prototype stress induced kinase, in which serine phosphorylates many signaling proteins including Insulin Receptor Substrate-1 (IRS-1) and IRS-2. This leads to inhibition of insulin signaling and stimulation of expression of SOCS (Suppressor of Cytokine Signaling) proteins, which binds IRS-1 and IRS-2 and mediates their degradation. ${ }^{20}$ Inflammatory cytokines such as TNF- $\alpha$, IL- $1 \beta$ and IL- 6 also down regulate peroxisome proliferatoractivated receptor- $\gamma$ (PRAR- $\gamma$ ) expression. ${ }^{21}$ Interestingly, insulin is itself an inhibitor of acute phase protein synthesis ${ }^{22}$ and in animal model of diabetes, the acute phase response is increased by insulin deficiency. ${ }^{23}$ This indicates that there could be a positive feedback in type 2 diabetes whereby cytokine induced insulin resistance further augments the acute phase response. The relative normal levels of acute phase reactants in type 1 diabetes suggests that insulin replacement and much lesser degree of hepatic insulin resistance in this type of diabetes is sufficient to restrain acute phase protein production. In the short term, innate immunity has survival value and restores homeostasis after an environmental stress, but in type 2 and impaired glucose tolerance (IGT), it may be that prolonged lifestyle or environmental stimulants cause maladaptation to the normal psychological response to stress, causing disease instead of repair A genetic or inborn propensity to a hyper responsive innate immune system might exist in certain individuals. This notion is supported by recent evidence that disproportionate size at birth is associated elevated levels of acute phase reactants such as cortisol and fibrinogen in adult life. ${ }^{24}$

Insulin sensitivity or resistance are associated with specific polymorphisms in the TNF- $\alpha$ gene promoter, ${ }^{25} \mathrm{TNF}-\alpha$ receptor gene and IL-6 gene. ${ }^{26}$ Nondiabetic subjects with family history of type 2 diabetes have higher circulating CRP levels than age and BMI matched control subjects without a family history. ${ }^{27}$ Activation of innate immunity in the genetically and metabolically programmed individual, including the effects of fat and the $\mathrm{n} 3$ : $\mathrm{n} 6$ fatty acid ratio on cytokine production may contributed by many dietary factors. ${ }^{28}$ Adipose tissue IL-6 production is increased by some five folds after milk intake when measured by subcutaneous microperfusion, this offering a mechanism by which repeated dietary excess might favor hypercytokinemia. ${ }^{29}$ Vlassara et $a 1 .^{30}$ recently showed that administration of high advanced glycation end products (AGEs) diet to type 1 and type 2 diabetic subjects caused plasma CRP and mononuclear cell TNF- $\alpha$ to increase whereas a low AGEs diet caused CRP and TNF- $\alpha$ to decreased. ${ }^{30}$ Cytokines production from monocytes and macrophage and circulating acute phase protein, IL- 6 and TNF- $\alpha$ increase with age, as of course does the propensity to develop type 2 diabetes. Indeed it has been argued that a major characteristic of aging is a global reduction in the capacity to cope with a variety of stressors and a concomitant increase in proinflammatory status. ${ }^{31}$

A major uncertainty is whether hyperglycemia is a main determinant of the inflammation in type 2 diabetes mellitus- there is evidence for and against. Cross sectional studies of type 2 diabetes mellitus show that CRP and IL-6 are significantly correlated with blood glucose concentration or glycated hemoglobin percentage. ${ }^{32}$ Lowering of blood glucose levels in type 2 diabetic patient are accompanied by reduced levels of inflammatory markers. ${ }^{33}$ Recent finding indicates that acute hyperglycemia in nondiabetic and IGT subjects elevate plasma IL- 6 and TNF- $\alpha$ concentration higher and longer in an individual with IGT and when glucose was given as pulse. ${ }^{34}$ By infusion of the antioxidant glutathione the effect was abolished, which suggests that hyperglycemia induced cytokine production is mediated by reactive oxygen species. Subcutaneous and intraabdominal adipose tissue is a major source of TNF- $\alpha$ and IL- 6 production. This raise the question of whether the acute phase reaction of type 2 diabetes is mainly secondary to obesity. In a recent study in which a case and control subjects were matched by BMI and waist circumference, neither CRP nor IL-6, predicted the development of type 2 diabetes, although lowered level of adiponectin did. Thus a hypothesis was suggested that because inflammatory markers are associated with obesity, they only indirectly predict diabetes and act as surrogate markers of hypoadeponectinemia. ${ }^{35}$ Having demonstrated that there is an inflammatory process going on in type 2 diabetes, we next thought of estimating inflammatory markers in patients on treatment( for at least 5 years) with oral hypoglycemic drugs. Many of the drugs have been shown to have anti-inflammatory effects. Statin drugs inhibits HMG-CoA reductase and prevent atherosclerosis and inhibit the acute phase response by diminishing the deposition of low density lipoprotein (LDL) particles rich in cholesterol and phospholipids in macrophages and smooth muscle cells. Statins were found to reduce CRP levels and did not correlate with the reduction of the lipid levels suggesting that the in addition to their ability to reduce LDL, statins may also inhibit the acute phase response. Freeman DJ et al. ${ }^{36}$ showed that statin therapy also prevent diabetes mellitus. Pravastatin therapy in the West of Scotland Coronary Prevention Study resulted in a 30\% reduction of risk of developing type 2 diabetes. ${ }^{36}$

Salicylates in high doses have been known to lower glycosuria in diabetic patients. Although earlier studies were contradictory, these studies has used lower aspirin doses $(<3 \mathrm{gm} /$ day $)$ and therapeutic duration was only for a few days. Hundal $\mathrm{RS}^{37}$ reported that high doses of aspirin (7 gm/day) for 2 wks caused $25 \%$ reduction in fasting plasma glucose, $50 \%$ reduction in triglyceride and $15 \%$ reduction of 
CRP concentration independently of the changes in the plasma insulin concentration. ${ }^{37}$ The other widely used oral hypoglycemic agents thiazolidinedions (Glitazone) are peroxizome proliferators activated receptor $\gamma$ ( PRAR $\gamma$ ) agonist that have been regarded as insulin sensitizes through mechanisms such as altered transcription of insulin sensitive genes controlling lipogenesis, adipocytes differentiation, fatty acid uptake and GLUT 4 ( Glucose Transporter 4) expression. They also have an anti-inflammatory action inhibiting cytokine production, macrophage activation and reducing CRP as well as WBC count in type 2 diabetic subjects. ${ }^{38}$ Angiotensin Converting Enzyme Inhibitors (ACE inhibitors) are also known to decrease insulin resistance in either type 1 or type 2 diabetic patients with concomitant hypertension. Torlone E et al..$^{39}$ demonstrated improved glycemic control in patients with arterial hypertension and type 2 diabetes using ACE inhibitors. Insulin has a potent anti-inflammatory activity. Insulin was found to be a rapid nonspecific and dose dependent inhibitors of the cytokine and glucocorticoids stimulation of acute phase protein, gene expression and exerted effect at the transcriptional levels. Insulin inhibition applied to all cell cytokines tested but to various degrees depending upon the particular acute phase gene. ${ }^{39}$ In this study, of the 25 type 2 diabetic patients on treatment for at least $5 \mathrm{yrs}, 8$ patient were in sulfonylurea-metformin combination, 7 were on glitazone, 6 were on sulfonylurea alone, 2 were on glitazone-metformin combination and 2 were on metformin alone. Metformin and other biguanides are derived from French lilac (Galega officinalis), and since the $1950 \mathrm{~s}$, metformin is the first choice in the oral treatment of T2DM. ${ }^{40}$ Several mechanisms of the glucose-lowering effect of metformin have been proposed including the generally accepted activation of AMP-activated protein kinase (AMPK), a cellular energy sensor activated under metabolic stress. Metformin not only improves hyperglycemia and insulin resistance but also has been shown to have anti-inflammatory, anticancer, and anti aging effects and to improve other cardiovascular risk factors, such as an overweight state or obesity, atherogenic dyslipidemia, blood pressure, procoagulant state, and carotid intima-media thickness.

Sulfonylureas target the ATP-sensitive potassium (KATP) channel to stimulate insulin secretion from pancreatic $\beta$-cells and are widely used in the treatment of Type 2 diabetes mellitus. ${ }^{41}$ Thiazolidinedione (TZDs) is a peroxisome proliferator-activated receptors activator (mainly PPAR- $\gamma$ ), have potential implications in molecular pathways of insulin resistance, type 2 diabetes Mellitus, and atherosclerosis. Rosiglitazone and pioglitazone, also known as TZDs, are selective agonists of nuclear transcription factor peroxisome proliferatoractivated receptor- $\gamma$ (PPAR- $\gamma){ }^{42}$ When compared with newly diagnosed untreated group (Group I) with treated group(Group II) the levels of $\alpha 1$-antitrypsin, $\alpha 1$-acid glycoprotein and ceruloplasmin were statistically lower (Table 5). No significant difference was found in the fibrinogen levels. The values of $\alpha 1$-acid glycoprotein and ceruloplasmin were comparable to those of the control group. The RBS values were similar to those of untreated group $(193.62 \pm 33.65$ and $193.26 \pm 35.30)$. It is interesting to note that $\alpha 1$-acid glycoprotein levels were almost the same in type 2 untreated patients and control (Table 3 ). Probably $\alpha 1$-acid glycoprotein is the most amenable acute phase protein to treatment modalities. Comparable ceruloplasmin levels in type 2 patients on treatment and controls again raise the question as to the 'prooxidant' or 'antioxidant' action of ceruloplasmin. No change in fibrinogen values suggest multiple pathway involvement that are poorly understood.

\section{Conclusion}

Currently, the prevalence of obesity and Type 2 Diabetes Mellitus has reached epidemic proportions throughout the world particularly in developed and some developing countries. Since cardio-metabolic abnormalities can be lead by type 2 diabetes mellitus, there is a clear need to address this worrying trend. Obesity and Type 2 Diabetes Mellitus cause an increase in inflammatory markers (CRP, TNF- $\alpha$, IL6) and a decrease in anti-inflammatory factors leading to metabolic dysfunction. Thus, it's very important and appropriate needs for targeting inflammation for the management of diabetes and related metabolic disorders. Multiple studies have demonstrated that various hypoglycemic drugs have anti-inflammatory potential, which can contribute to improved clinical outcomes. Hypoglycemic agents exert their anti-inflammatory effects either by controlling hyperglycemia or directly, by acting on inflammatory pathways, which is not dependent by control of glucose.

\section{Acknowledgements}

None.

\section{Conflict of interest}

Authors declare there is no conflict of interest.

\section{Funding}

This research did not receive any specific grant from funding agencies in the public, commercial, or not-for-profit sectors.

\section{References}

1. Centers for Disease Control and Prevention. Diabetes Report Card 2014 Atlanta GA. Centers for Disease Control and Prevention, US Dept of Health and Human Services, 2015.

2. Fausi AS, Braunwald E, Isselbacher KJ, et al. Endocrinology and metabolism, Diabetes Mellitus. 14th ed. In: Harrison's Principle of Internal Medicine. USA: Mc Graw Hill; 1998.

3. Luft VC, Schmidt MI, Pankow JS, et al. Chronic inflammation role in the obesity-diabetes association: a case-cohort study. Diabetol Metab Syndr. 2013;5(1):31

4. Esser N, Legrand-Poels S, Piette J, et al. Inflammation as a link between obesity, metabolic syndrome and type 2 diabetes. Diabetes Res Clin Pract. 2014;105(2):141-150.

5. Wellen KE, Hotamisligil GS. Inflammation, stress, and diabetes. J Clin Invest. 2005;115(5):1111-1119.

6. Pickup JC. Inflammation and activated innate immunity in the pathogenesis of type 2 diabetes. Diabetes care. 2004;27(3):813-823.

7. Pickup JC, Crook MA, Chusney GD, et al. NIDDM as a disease of the innate immune system: association of acute-phase reactants and interleukin- 6 with metabolic syndrome X. Diabetologia. 1997;40(11):1286-1292.

8. Snijder MB, Dekker JM, Visser M, et al. C-reactive protein and diabetes type 2. Diabetologia. 2001;44(1):115A.

9. Spranger J, Kroke A, Möhlig M, et al. Inflammatory cytokines and the risk to develop type 2 diabetes: results of the prospective population based European Prospective Investigation Cancer and Nutrition (EPICN)Potsdam study. Diabetes. 2003;52(3):812-818.

10. Garber AJ, Abrahamson MJ, Barzilay JI, et al. AACE/ACE comprehensive diabetes management algorithm. Endocr Pract. 2015;21(4):438-447.

11. Standards of medical care in diabetes-2015: summary of revisions. Diabetes Care. 2015;38(1):4 
12. DeFronzo RA. From the triumvirate to the ominous Octet: a new paradigm for the treatment of type 2 diabetes mellitus. Diabetes. 2009;58(4):773-795.

13. Fonseca VA. Defining and characterizing the progression of type 2 diabetes. Diabetes Care. 2009;32(2):S151-156.

14. Bloomgarden ZT, Handelsman Y. Approaches to treatment 2 comparison of AACE and ADA type 2 diabetes treatment guidelines. $J$ Diabetes. $2015 ; 8(1): 4-6$

15. Trinder P. A Simple Turbidimetric Method for the Determination of Serum Cholesterol. Ann Cli Biochem. 1969;6:165.

16. Varley H, Gowenlock AH, Bell M. Determination of plasma fibrinogen. In; Practical clincial biochemistry. 5th ed. CBS publishers and distributors; 1991. p. 557-559.

17. Sunderman FW, Nomoto S. Measurement of human serum ceruloplasmin by its p-phenylenediamineoxi-dase activity. Clin Chem.1970;16(11):90-910.

18. Sundaresh CS, Aroor AR, Pattabiraman TN. Compara-tive study of amidolytic and caseinolytic methods for the determination of urinary trypsin inhibitor. Indian J Med Res. 1978;68:341-347.

19. Winzler RJ. Determination of serum $\alpha-1$ acid gly-coprotein. In: Methods in Biochemical Analysis. New York: Inter-science Pub; 1955:270.

20. Medzhitov R, Janeway C. Innate immunity. NEngl J Med. 2000;343(5):338344.

21. Fearon DT. Seeking wisdom in innate immunity. Nature. 1997;388(6640):323-324.

22. Campus SP, Baumann H. Insulin is a prominent modulator of the cytokine stimulated expression of acute phase plasma protein genes. Mol Cell Biol. 1992;12(4):1789-1797.

23. Pickup JC, Day C, Bailey CJ, et al. Plasma sialic acid in animal models of diabetes mellitus: evidence of modulation of sialic acid concentrations by insulin deficiency. Life Sci. 1995;57(14):1383-1391.

24. Phillips DI, Walker BR, Reynolds RM, et al. Low birth weight predicts elevated plasma cortisol concentration in adults from 3 population. Hypertension. 2000;35(6):1301-1306.

25. Day CP, Grove J, Daly AK, Stewart MW, et al. Tumor necrosis factor alpha gene promoter polymorphism and decreased insulin resistance. Diabetologia. 1998;41(4):430-434.

26. Fernandez-Real JM, Broach M, Vendrell J, et al. Interleukin-6 gene polymorphism and insulin sensitivity. Diabetes. 2000;49(3):517-520.

27. Pannacciuli N, De Pergola G, Giorgino F, et al. A family history of type 2 diabetes is associated with increased plasma levels of C-reactive protein in non-smoking healthy women. Diabet Med. 2002;19(8):689-692.

28. Endres S, Ghorbani R, Kelley VE, et al. The effect of dietary supplementation with $\omega-3$ polyunsaturated fatty acids on the synthesis of interleukin-1 and tumor necrosis factor by mononuclear cells. $N$ Engl $J$ Med. 1989;320(5):265-271.
29. Orban Z, Ramaley AT, Sampson M, et al. The differential effect of food intake and $\beta$-adrenergic stimulation on adipose derived hormones and cytokines in man. J Clin Endocrinol Metab. 1999;84(6):2126-2133

30. Vlassara HC, Crandall J, Goldberg T, et al. Inflammatory mediators are induced by dietary glycotoxins, a major risk factor for diabetic angiopathy. Proc Natl Acad Sci USA. 2002;99(24):15596-15610.

31. Francessi C, Bonate M, Valensin S, et al. Inflamm-aging: an evolutionary perspective on immunosenescence. Ann NY Acad Sci. 2000;908:244-254.

32. Rodriguez-Moran M, Guerrero-Romero F. Increased level of C-reactive protein in noncontrolled type 2 diabetic subjects. J Diabetes Complications. 13(4):211-215.

33. Ceriello A, Mercuri F, Fabbro D, et al. Effects of intensive glycemic control on fibrinogen plasma concentration in patient with type 2 diabetes: relation with $\beta$-fibrinogen genotype. Diabetologia. 1998;41(11):1270-1273.

34. Esposito K, Nappo F, Marietta R, et al. Inflammatory cytokines concentration are acutely increased by hyperglycemia in humans: role of oxidative stress. Circulation. 2002;106(16):2067-2072.

35. Krakott J, Funahashi T, Slehouwer CD, et al. Inflammatory markers, adiponectin and risk of type 2 diabetes in the Pima Indian. Diabetes care. 2003;26(6):1745-1751.

36. Freeman DJ, Norrie J, Satter N. Pravastatin and the development of diabetes mellitus: evidence for a protective treatment in the West of Scotland Coronary Prevention Study. Circulation. 2001;103(3):357-362.

37. Hundal RS, Petersen KF, Mayerson AB, et al. Mechanism by which high dose aspirin improves glucose metabolism in type 2 diabetes. $J$ Clin Invest. 2002;109(10):1321-1326.

38. Ricole M, Li AC, Willison TM, et al. The peroxizome proliferators activated receptor(gamma) is a negative regulator of macrophage activation. Nature. 1988;391(6662):79-82.

39. Torlone E, Britta M, Rambotti AM, et al. Improved glycemic control after long term angiotensin converting enzyme inhibition in subjects with arterial hypertension and type 2 diabetes. Diabetes Care. 1993;16(10):1347-1352.

40. Saisho Y. Metformin and inflammation: its potential beyond glucoselowering effect. Endocr Metab Immune Disord Drug Targets. 2015;15(3):196-205.

41. Proks P, Reimann F, Green N, et al. Sulfonylurea stimulation of insulin secretion. Diabetes. 2002;51(3):S368-376.

42. Corzo C, Griffin PR. Targeting the peroxisome proliferator-activated receptor- $\gamma$ to counter the inflammatory milieu in obesity. Diabetes Metab J. 2013;37(6):395-403. 